\title{
Aggregation is a critical cause of poor transfer into the brain tissue of intravenously administered cationic PAMAM dendrimer nanoparticles
}

This article was published in the following Dove Press journal:

International Journal of Nanomedicine

24 May 2017

Number of times this article has been viewed

\author{
Yoshika Kurokawa' \\ Hideko Sone' \\ Tin-Tin Win-Shwe' \\ Yang Zeng' \\ Hiroyuki Kimura ${ }^{2}$ \\ Yosuke Koyama' \\ Yusuke Yagi² \\ Yasuto Matsui ${ }^{3}$ \\ Masashi Yamazaki ${ }^{4}$ \\ Seishiro Hirano' \\ 'Center for Health and Environmenta \\ Risk Research, National Institute \\ for Environmental Studies, Tsukuba, \\ Ibaraki, ${ }^{2}$ Department of Analytical \\ and Bioinorganic Chemistry, \\ Kyoto Pharmaceutical University, \\ ${ }^{3}$ Department of Environmental \\ Engineering, Kyoto University \\ Graduate School of Engineering, \\ Kyoto, ${ }^{4}$ TIA Center Office, National \\ Institute of Advanced Industrial \\ Science and Technology, Tsukuba, \\ Ibaraki, Japan
}

Correspondence: Yoshika Kurokawa Center for Health and Environmental Risk Research, National Institute for Environmental Studies, Onogawa 16-2, Tsukuba, Ibaraki 305-8506, Japan Tel +8I 0298502437

Email kurokawa@nies.go.jp

\begin{abstract}
Dendrimers have been expected as excellent nanodevices for brain medication. An amine-terminated polyamidoamine dendrimer (PD), an unmodified plain type of $\mathrm{PD}$, has the obvious disadvantage of cytotoxicity, but still serves as an attractive molecule because it easily adheres to the cell surface, facilitating easy cellular uptake. Single-photon emission computed tomographic imaging of a mouse following intravenous injection of a radiolabeled PD failed to reveal any signal in the intracranial region. Furthermore, examination of the permeability of PD particles across the blood-brain barrier (BBB) in vitro using a commercially available kit revealed poor permeability of the nanoparticles, which was suppressed by an inhibitor of caveolae-mediated endocytosis, but not by an inhibitor of macropinocytosis. Physicochemical analysis of the PD revealed that cationic PDs are likely to aggregate promptly upon mixing with body fluids and that this prompt aggregation is probably driven by non-Derjaguin-LandauVerwey-Overbeek attractive forces originating from the surrounding divalent ions. Atomic force microscopy observation of a freshly cleaved mica plate soaked in dendrimer suspension (culture media) confirmed prompt aggregation. Our study revealed poor transfer of intravenously administered cationic PDs into the intracranial nervous tissue, and the results of our analysis suggested that this was largely attributable to the reduced BBB permeability arising from the propensity of the particles to promptly aggregate upon mixing with body fluids.
\end{abstract}

Keywords: PAMAM dendrimer, nanomaterial, aggregation, blood-brain barrier

\section{Introduction}

Artificial nanomaterials have been considered to hold high promise as components of multipurpose medical devices, particularly in brain medication. ${ }^{1}$ Attempts have been made in the field of nanomedicine to design nanomaterials as carriers of various drugs, including anticancer, antimicrobial, and scavenging drugs, as carriers of DNA/RNA for the therapy of several congenital hereditary diseases, and as an image enhancer for several types of bioimaging. In general, in relation to nanodevice-implemented delivery of biomaterials, a number of technical difficulties need to be overcome, such as how the materials can escape the clearance systems of the living body, how they can be guided to the target organs, how they can be internalized and utilized in the target cells, and how adverse effects associated with their use can be avoided. ${ }^{2}$ The second of the above items, namely, targeting, is a special problem when the target organ is the brain because of the existence of the blood-brain barrier (BBB). ${ }^{3}$

Dendrimers are nanoparticles with unique features: they are usually spherical in shape, show wide variances of size and surface radicals, and contain an inner pocket. ${ }^{4}$ They are built of a series of branches from core element and are utilized as binders 
or containers of several other materials. ${ }^{5}$ Dendrimers have so far been considered for various medical applications, in particular, as carriers of microRNAs in gene therapy, ${ }^{6}$ as components of macromolecular micelles in drug delivery system, ${ }^{7}$ and as magnetic resonance imaging contrast agents in the gadolinium-bound form. ${ }^{8}$ Polyamidoamine (PAMAM) dendrimers are the prototype of dendrimer particles and were the first dendrimer molecules synthesized by Tomalia et al in $1985 .^{9}$ The original type of PAMAM dendrimer has an overall surface of amine terminates, and the amines confer a large binding capacity for various molecules. Owing to the cationic surface, amine-terminated PAMAM dendrimer particles tend to be easily adsorbed on to cell membranes and are consequently internalized promptly by the cells and trapped within endosomal vesicles, finally succumbing to lysosomal digestion. ${ }^{10}$ However, PAMAM dendrimer particles have many tertiary amines within the particles, and these amines act as buffers against invasion by protons outside the lysosomes, aiding in their lysosomal escape, which process is called "proton sponge effect". ${ }^{11,12}$ For all the merits of PAMAM dendrimer particles as nanocarriers to target cells, they have the disadvantage of exerting explicit cytotoxicity. ${ }^{13,14}$ Several modifications of the surfaces of PAMAM dendrimer particles have been attempted to achieve a lower cytotoxicity of these dendrimers, in exchange for a lower cellular uptake, and conjugation with polyethyleneglycol is currently considered as the most effective modification in this direction. ${ }^{15}$

Permeation across the $\mathrm{BBB}$ is a critical process for the translocation of nanoparticles into the brain tissue. PAMAM dendrimer particles are considered to face no counteracting antibodies, receptors, or transporter molecules on the cell surface, and the first process in their transport across the BBB is adhesion to the surface of the endothelial cells comprising the BBB. As unmodified plain PAMAM dendrimer particles may be advantageous in relation to BBB permeation owing to their cationic surface, they could serve as an attractive nanodevice for brain medication, provided their cytotoxicity could be suppressed locally in the cytosol region, where other studies have indicated that dendrimers exert their cytotoxicity by attacking mitochondria. ${ }^{16,17}$

This study was focused on the amine-terminated cationic PAMAM dendrimer. In this study, we examined the distribution of intravenously administered dendrimer particles to the brain in mice by in vivo single-photon emission computed tomographic (SPECT) imaging, and conducted physicochemical analyses and other experiments in vitro for clarifying factors that regulate the translocation of these particles to the brain. Our findings revealed poor transport of the intravenously administered dendrimer particles into the intracranial nervous tissue, largely attributable to the propensity of the cationic PAMAM dendrimer particles to form aggregates upon mixing with body fluids.

\section{Materials and methods Fluorescence labeling of the dendrimer}

The amine-terminated PAMAM dendrimer(1,4-diaminobutane core, generation 4) was purchased from Sigma-Aldrich Co. (St Louis, MO, USA). Alexa Fluor 488 or 546 of the succinimidyl ester type was purchased from Molecular Probes Inc. (Eugene, OR, USA).

Labeling was started by mixing the dendrimer $(2 \mathrm{mg})$ with Alexa Fluor $(0.2 \mathrm{mg})$ in $0.5 \mathrm{~mL}$ of $0.1 \mathrm{M}$ sodium bicarbonate solution ( $\mathrm{pH} \mathrm{8.3).} \mathrm{The} \mathrm{mixture} \mathrm{was} \mathrm{kept} \mathrm{in} \mathrm{mild} \mathrm{stirring} \mathrm{at}$ room temperature (RT) for 4 hours and then dialyzed against $1 \mathrm{~L}$ of pure water three times for removing the free molecules of Alexa Fluor. The dialysate was kept frozen $\left(-30^{\circ} \mathrm{C}\right)$ until the days of the experiments. The labeling efficiencies were calculated approximately as 1.5 mole Alexa Fluor 488 per mole dendrimer and 0.7 mole Alexa Fluor 546 per mole dendrimer.

\section{Radiolabeling of the dendrimer}

$\mathrm{N}$-succinimidyl 3-( $\left.{ }^{123} \mathrm{I}\right)$ iodobenzoate ( ${ }^{123} \mathrm{I}$-SIB) was prepared according to the method described by Vaidyanathan and Zalutsky. ${ }^{18} N$-succinimidyl 3-(tri- $n$-butylstannyl) benzoate (STB), synthesized preliminarily from 3-bromobenzoic acid, $n$-butyllithium, and tri- $n$-butyl tin chloride, was dissolved in $5 \%$ acetic acid/95\% methanol and then mixed with a solution of $\left({ }^{123} \mathrm{I}\right)$ ammonium chloride $(10 \mathrm{mCi})$. After stirring for 30 minutes at RT in the presence of $N$-chlorosuccinimide, the mixture was acidified to $\mathrm{pH} 2-3$ and subjected to high-performance liquid chromatography with COSMOSIL $5 \mathrm{C}_{18}$-AR-II (Nacalai tesque, Kyoto, Japan) and to solidphase extraction with Sep-Pak $\mathrm{C}_{18}$ (Waters, MA, USA) for the purpose of purifying ${ }^{123} \mathrm{I}-\mathrm{SIB}$. The ${ }^{123} \mathrm{I}-\mathrm{SIB}$ solution and the suspension of PAMAM dendrimer $(5 \mathrm{mg}, 50 \mu \mathrm{L})$ were mixed at $\mathrm{pH} 9$, and after 1 hour, the mixture was neutralized, concentrated, and finally diluted with normal saline. The isolated yield of radiolabeled PAMAM dendrimer was $21 \%$ (2.1 mCi). The radiochemical purity, determined by radio-thin-layer chromatography $(\mathrm{TLC})(\mathrm{MeOH}$ : water $=1: 1)$, was $>95 \%$.

\section{SPECT imaging}

A 4-week-old male ddY mouse weighing $23 \mathrm{~g}$ was purchased from Japan SLC (Shizuoka, Japan). The study was 
conducted in conformity with the guidelines for the use and care of laboratory animals established by the Science Council of Japan and approved by the Kyoto University Animal Care Committee. SPECT/computed tomography (CT) was performed using the Triumph LabPET12/SPECT4/CT (TriFoil Imaging Inc., Chatsworth, CA, USA). The mouse was given an intravenous injection of the radiolabeled dendrimer suspension $(500 \mu \mathrm{Ci}, 50-70 \mu \mathrm{L})$, and at 1,3 , and 7 hours after the injection, the mouse was anesthetized with $2 \%$ isoflurane and placed on the scanner bed. Tomographic spiral SPECT scans were acquired for 20 minute using a 4-head detector camera, followed by plain CT image acquisition for anatomic reference (spatial resolution, $50 \mu \mathrm{m} ; 60 \mathrm{kV} ; 310 \mu \mathrm{A}$ ). In the SPECT studies, all projection data were acquired through single-pinhole collimators $(1.0 \mathrm{~mm}$ diameter, $9.0 \mathrm{~mm}$ focal length) using a $20 \%$ energy window centered on $159 \mathrm{keV}$ for ${ }^{123} \mathrm{I}$, a $35-\mathrm{mm}$ radius of rotation (ROR), a $360^{\circ}$ circular orbit, and a $60 \mathrm{~s} / 32$ angles projection. The SPECT images and CT images were reconstructed by the three-dimensional (3D) ordered-subsets expectation maximization algorithm (five iteration and eight subsets) and the modified 3D cone-beam Feldkamp algorithm, respectively. The acquired data sets of SPECT and CT were processed with the FEI Amira software (version 5.1) (FEI, Hillsboro, OR, USA).

\section{BBB permeability test}

The BBB permeability of the dendrimer particles was evaluated by a commercially available in vitro test kit (RBT-24H, PharmaCo-Cell Co. Ltd., Nagasaki, Japan). Before the assays, the transendothelial electrical resistance (TEER) of the BBB was measured with a TEER measurements system (MillicellERS, Millipore Inc., Billerica, MA, USA) and the value was confirmed to be $>150 \Omega \cdot \mathrm{cm}^{2}$. After exchanging the culture medium for an assay (phosphate buffered saline with calcium and magnesium (PBS(+)), 0.01 M (4-[2-hydroxyethyl]1-piperazineethanesulfonic acid, $4.5 \mathrm{mg} / \mathrm{mL}$ D-glucose), the Alexa 488-labeled dendrimer particles were added to the apical aspect of the BBB layer. After 30-minute incubation with mild shaking, the fluorescence intensities of the assay buffer on the basolateral aspect of the BBB were measured. The permeability coefficient $\left(P_{\text {app }}[\mathrm{cm} / \mathrm{s}]\right)$ was calculated using the following equation:

$$
P_{\text {app }}=V \frac{\Delta[C]_{b}}{\Delta t} \frac{1}{A[C]_{a}}
$$

where $V$ and $A$ denote the medium volume in the basolateral aspect $\left(\mathrm{cm}^{3}\right)$ and the surface area of the membrane $\left(\mathrm{cm}^{2}\right)$, respectively, and $\Delta[C]_{\mathrm{b}}$ and $[C]_{\mathrm{a}}$ denote the differential concentration of the particles in the basolateral aspect $\left(\mathrm{mL}^{-1}\right)$ and the initial concentration of the particles in the apical aspect $\left(\mathrm{mL}^{-1}\right)$, respectively.

In the tests for examining the effects of addition of various drugs (cell uptake inhibitors) to the assay buffer, the experiment was preceded by incubation of the assay buffer with the subject drug alone for 30 minutes.

\section{Measurement of dynamic light scattering}

At first, the viscosities of the sample suspensions were measured with a viscometer (SV-1A, A\&D Co. Ltd, Tokyo, Japan). Measurement of the dynamic light scattering (DLS) was carried out with a particle size analyzer (ELSZ-2, Otsuka Electronics Co. Ltd., Osaka, Japan). A light beam of a semiconductor laser $(\lambda=660 \mathrm{~nm})$ was directed at the suspensions, and the intensity of light scatter at an angle of $165^{\circ}$ was monitored. The autocorrelation of the time series was calculated, and by fitting the data to an exponential curve using the Levenberg-Marquardt algorithm, the dilution coefficient of the particles was estimated for calculating the particle size by the Stokes-Einstein equation. The measurement was repeated 70 times and finally a histogram of the particle size distribution was constructed.

\section{Fluorescence correlation spectroscopy}

Fluorescence correlation spectroscopy (FCS) was conducted with a confocal laser-scan inverted microscope (FV1200, Olympus, Tokyo, Japan). A glass cylinder (inner diameter: $4 \mathrm{~mm}$; height: $8 \mathrm{~mm}$ ) attached to a glass-bottom culture dish (diameter: $35 \mathrm{~mm}$ ) was filled with a suspension $(50 \mu \mathrm{L})$ of the fluorescence-labeled dendrimer. A fixed laser beam was applied to the sample through a water-immersion $40 \times$ objective lens (UAPON40XW340, Olympus) and the fluorescent light was detected with a high-sensitive photodetector (GaAsP PMT, Olympus), which counted photons at intervals of $10 \mu \mathrm{s}$ for $40 \mathrm{~s}$. The autocorrelation was calculated from the time series data and the result was fitted, using the Levenberg-Marquardt algorithm, to the curve using the following equation:

$$
g(\tau)=1+\left(1+\frac{a}{1-a}\right) \exp \left(-\frac{\tau}{b}\right) \frac{1}{N} \frac{1}{\left(1+\frac{4 D \tau}{\omega_{0}^{2}}\right) \sqrt{1+\frac{4 D \tau}{\omega_{z}^{2}}}}
$$

where $a$ and $b$ are parameters for triplet blinking (proportion of fraction entering the triplet state and triplet state relaxation 
time, respectively), $N$ and $D$ are particle parameters (average number in confocal volume and diffusion coefficient), and $w_{0}$ and $w_{\mathrm{z}}$ are optical parameters (radial and axial radii of the confocal volume). The values of $w_{0}$ and $w_{z}$ in the above equation were estimated by preliminary measurement in a single suspension of Alexa 546 molecules.

\section{Calculation of the critical coagulation concentration}

If dendrimer nanoparticles in liquid phase are assumed to interact with each other simply by two physical forces, that is the electrostatic repulsive force and the van der Waals attractive force, the dynamics of dispersion or aggregation of the particles can be formularized based on the DerjaguinLandau-Verwey-Overbeek (DLVO) theory. According to this, the critical coagulation concentration (CCC) (mM) of the electrolytes in nanoparticle suspension, namely, the minimum concentration of electrolytes for inducing prompt aggregation of the suspended particles, can be calculated using the following equation:

$$
C C C=\frac{73728 \pi^{2} k^{5}\left(\varepsilon_{0} \varepsilon_{r}\right)^{3} \gamma^{5}}{\exp (2) e_{0}^{6} N_{A} A^{2} z^{6}} \tanh ^{4}\left(\frac{e_{0} z \psi_{0}}{4 k T}\right)
$$

where $k, \varepsilon_{0}, e_{0}$, and $N_{A}$ are physical constants (Boltzmann constant, dielectric constant, elementary electric charge, and Avogadro constant), and $T, \varepsilon_{r}$, and $z$ are parameters of the dispersion medium (liquid temperature, relative dielectric constant, and charge value of the electrolyte ions), and $\psi_{0}$ and $A$ are particle parameters (surface electric potential and Hamaker constant). The zeta potential (mV) of dendrimer was measured with DelsaMax PRO (Beckman Coulter Inc., Brea, CA, USA), and the value was substituted for $\psi_{0}$.

\section{Atomic force microscopy}

All images were acquired using the SPM-9700 (Shimadzu, Kyoto, Japan) in the clean room regulated at $22.5^{\circ} \mathrm{C} \pm 2{ }^{\circ} \mathrm{C}$. Original dendrimer suspension $(100 \mathrm{mg} / \mathrm{mL})$ in methanol was diluted with three-fold volume of culture media, Dulbecco's Modified Eagle's Medium (DMEM), and soon $1 \mu \mathrm{L}$ of the mixture was dropped onto a freshly cleaved 10-mm square mica plate fixed on a petri dish in the SPM stage. Immediately the dish was filled with $400 \mu \mathrm{L}$ of DMEM, and several minutes later, atomic force microscopy (AFM) observation was performed in liquid using a $125 \mu \mathrm{m}$ n-doped silicon cantilever (BL-AC40TS-C2, Olympus, Tokyo, Japan) with typical spring constants of $0.1 \mathrm{~N} / \mathrm{m}$ and typical resonance frequency of $25 \mathrm{kHz}$ (in water). Scanning the mica plate in DMEM only was performed beforehand to confirm the absence of any adsorbed materials, and selected fields of the mica in dendrimer suspension were scanned at rates of $2.0 \mathrm{~Hz}$.

\section{Results \\ SPECT imaging}

Figure 1 shows the results of the SPECT imaging conducted after intravenous injection into a mouse of ${ }^{123}$ I-labeled PAMAM dendrimer particles. At 1, 3, and 7 hours after the injection, radioactive signals were detected in the liver, spleen, and kidney, but not in the intracranial region. The high degree of distribution in the kidney, liver, and spleen indicated the remarkable removal of the particles from the blood circulation by renal glomerular filtration and trapping by the reticuloendothelial system (RES).

\section{BBB permeability test}

We estimated the BBB permeability of the dendrimer particles by applying a suspension of Alexa 488-labeled dendrimer particles $(1 \mu \mathrm{g} / \mathrm{mL})$ to the BBB kit, and the $P_{\text {app }}$ of the dendrimer particles was calculated as $8.0 \times 10^{-6} \mathrm{~cm} / \mathrm{s}$. Next, we added three kinds of cell uptake inhibitors to the assay solution: chlorpromazine $(10 \mu \mathrm{g} / \mathrm{mL})$, an inhibitor of clathrinmediated endocytosis; genistein $(200 \mu \mathrm{M})$, an inhibitor of caveolae-mediated endocytosis; and 5-( $N$-ethyl- $N$-isopropyl) amiloride (EIPA) $(50 \mu \mathrm{M})$, an inhibitor of macropinocytosis. The results revealed significant suppression of the dendrimer permeation by genistein, but not by chlorpromazine or EIPA (Figure 2), suggesting that a small number of dendrimer particles undergo transcytosis through the cells comprising the $\mathrm{BBB}$ via caveolae-mediated endocytosis.

\section{Determination of the particle size distribution by the DLS}

DLS measurements were conducted in samples of dendrimer particles suspended in various buffer solutions $(100 \mathrm{mg} / \mathrm{mL})$. The particles suspended in PBS (-) maintained their dispersed condition (diameter 4-5 nm) semi-permanently (Figure 3A). In contrast, the particles suspended in PBS $(+)$, or in various cell culture media, such as DMEM, showed marked aggregation (Figure 3B). For particles suspended in human serum albumin (HSA, $50 \mathrm{mg} / \mathrm{mL}$ )/PBS (-) or PBS (+), the peak 4-5 nm size distribution diminished or disappeared, but to which portion the size distribution of the dendrimers shifted was not clear (Figure 3C and D).

Under stepwise addition of sodium chloride to a dendrimer suspension in $\mathrm{H}_{2} \mathrm{O}$, aggregation was entirely suppressed, even when the $\left[\mathrm{Na}^{+}\right]$reached $380 \mathrm{mM}$. However, under stepwise addition of calcium chloride to a dendrimer suspension in $\mathrm{H}_{2} \mathrm{O}$, prompt aggregation occurred even when 
A

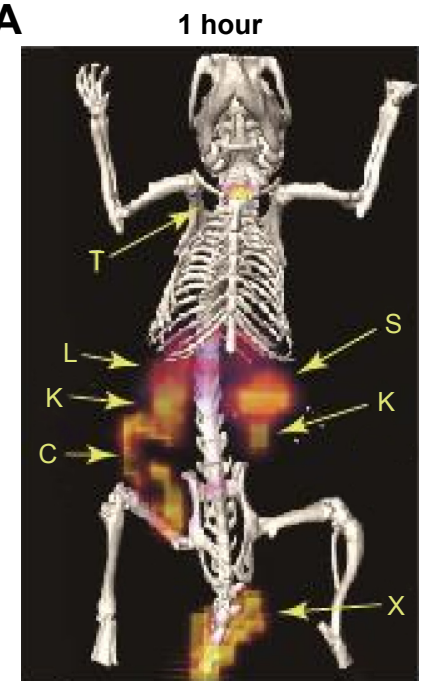

50
3 hours

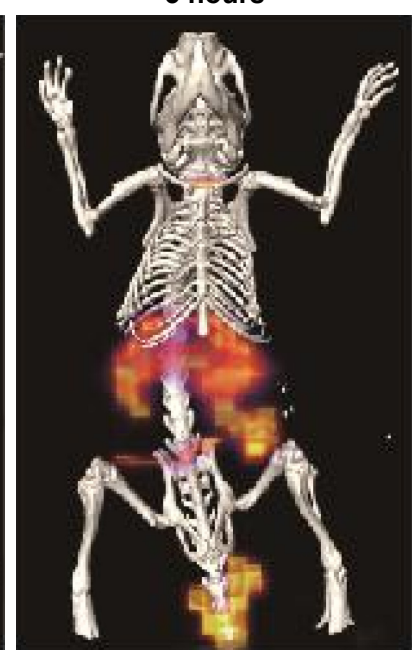

3 hours

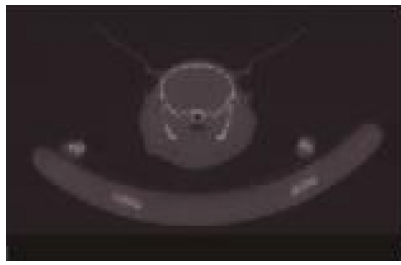

7 hours

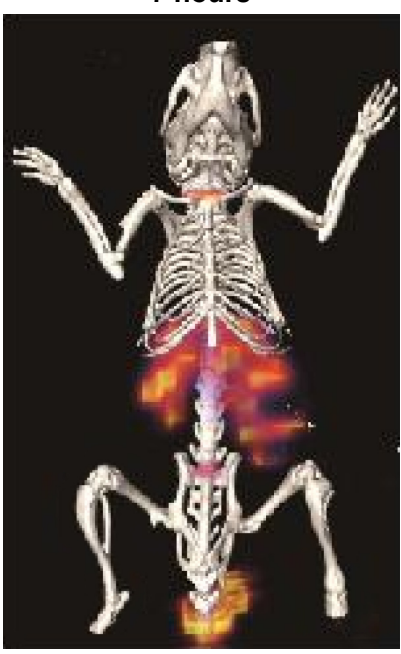

250

B

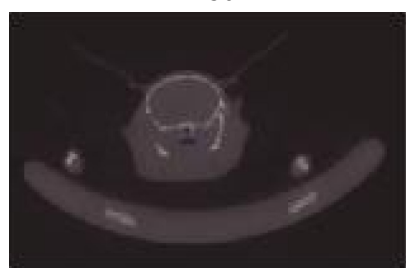

1 hour

0
7 hours

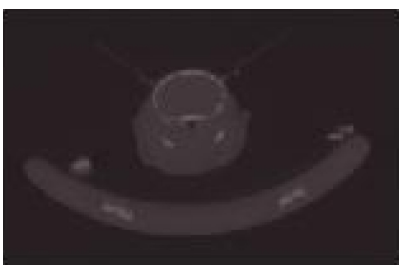

150

Figure I SPECT/CT images of a mouse at I, 3, and 7 hours after intravenous injection of ${ }^{123}$-labeled cationic PAMAM dendrimers.

Notes: (A) Coronal-view images after fusion of SPECT images with maximum intensity projection and CT images with surface rendering. (B) Transverse plane images along the cranial central portion.

Abbreviations: C, colon; CT, computed tomography; I, iodine; K, kidney; L, liver; PAMAM, polyamidoamine; S, spleen; SPECT, single-photon emission computed tomography; $\mathrm{T}$, thyroid; $\mathrm{X}$, injected portion.

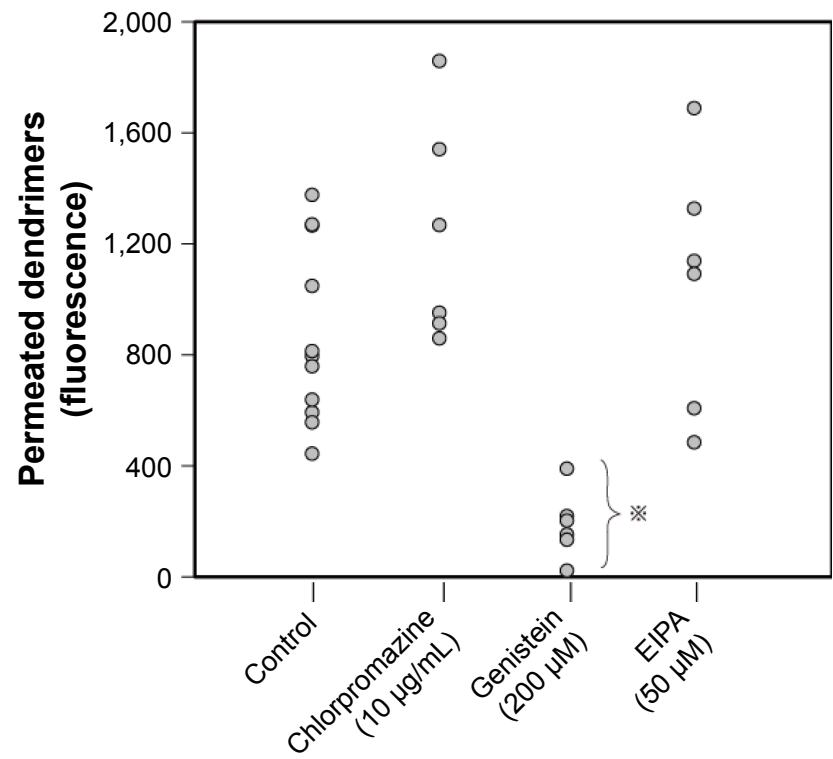

Figure 2 Results of the blood-brain barrier permeation test using Alexa 488-labeled cationic PAMAM dendrimer particles under pretreatment with various cellular uptake inhibitors.

Note: $※$ Significantly different $(P<0.05)$ from the control data as determined by Dunnett's test.

Abbreviations: EIPA, 5-( $N$-ethyl- $N$-isopropyl) amiloride; PAMAM, polyamidoamine. the $\left[\mathrm{Ca}^{2+}\right]$ reached just $1.0 \mathrm{mM}$, that is, almost equivalent to the concentration in PBS $(+)$ (data not shown).

\section{Determination of the particle size by FCS} As DLS measurement could not clarify the effect of HSA in the dendrimer suspension (Figure 3C and D), we performed FCS using Alexa 546-labeled dendrimer particles. Figure 4A shows the autocorrelation curve for a simple suspension of dendrimer particles and the particle diameter was estimated to be $\sim 4 \mathrm{~nm}$. Figure 4B shows the correlation curve for a dendrimer suspension in HSA/PBS (-) and the particle diameter was estimated to be $\sim 10 \mathrm{~nm}$. Furthermore, the ratio of the average fluorescence intensity of the suspension to the estimated value of $N$ in equation 2, which represents the average fluorescence intensity of the fluorescent particles in a confocal volume, was almost the same for the dendrimer particles suspended in water as that for the dendrimer particles suspended in HSA/PBS (-). These results suggest that each particle in the HSA/PBS (-) suspension represented a complex of a single HSA molecule and a single dendrimer 

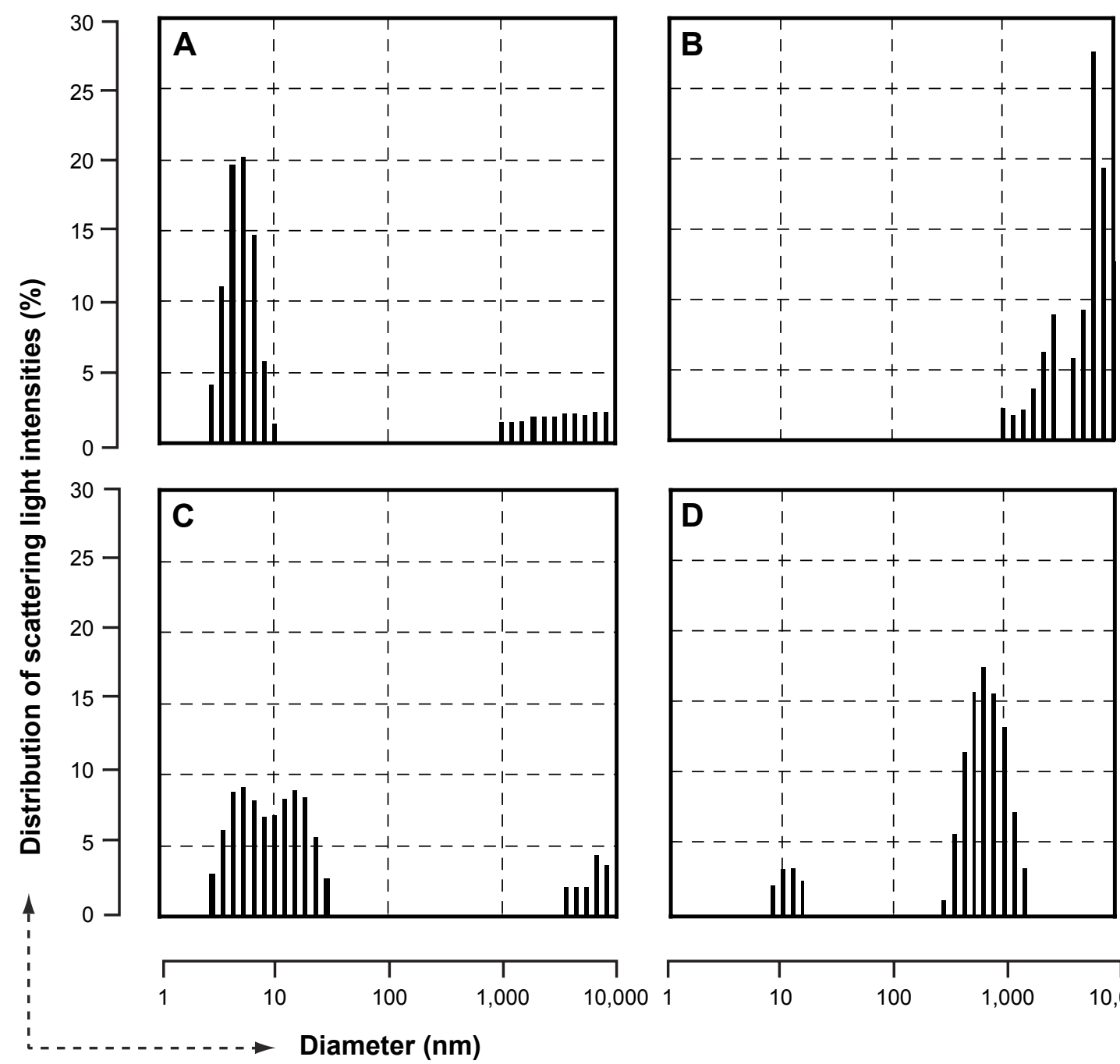

Figure 3 Histograms of particle size distribution determined by dynamic light scattering.

Notes: Cationic PAMAM dendrimers were suspended in the following solutions: (A) PBS (-); (B) PBS (+); (C) PBS (-) with HSA (50 g/mL); (D) PBS (+) with HSA (50 g/mL). The dispersed primary particles of the dendrimer were distributed around a diameter of $4 \mathrm{~nm}$.

Abbreviations: HSA, human serum albumin; PAMAM, polyamidoamine; PBS(-), phosphate buffered saline without calcium/magnesium; PBS(+), phosphate buffered saline with calcium/magnesium.

molecule. FCS analysis of a suspension of the dendrimer particles in HSA/PBS (+) showed a large size of the fluorescent particles within the laser spot (Figure 4C).

\section{Calculation of the CCC in the dendrimer aggregates}

According to Equation 3, the Hamaker constant of a dendrimer can be derived as follows:

$$
A=\sqrt{\frac{73728 \pi^{2} k^{5}\left(\varepsilon_{0} \varepsilon_{r}\right)^{3} \gamma^{5}}{\exp (2) e_{0}^{6} N_{A} z^{6}(C C C)} \tanh ^{4}\left(\frac{e_{0} z \psi_{0}}{4 k T}\right)}
$$

We determined the $\psi_{0}$ value of the dendrimer as $21.5 \mathrm{mV}$ by measuring the zeta potential. The value of $T$ (temperature) was set within the range of 293-310 K, and under this condition, the $\varepsilon_{\mathrm{r}}$ (relative dielectric constant) was estimated as 78.0-74.4. When the value of CCC in $z=1$ was set at $380 \mathrm{mM}$, the value of the Hamaker constant of the dendrimer was roughly estimated

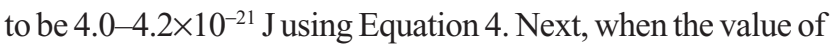
$A$ was set at $4.0-4.2 \times 10^{-21}$, the value of CCC in $z=2$ was found to be $\sim 80 \mathrm{mM}$, using Equation 3. As the $\mathrm{CCC}$ in $z=1 \mathrm{might}$ be $>380 \mathrm{mM}$ as suggested by the above-mentioned results of measurement of the DLS, the value of $A$ of the dendrimer might be $<4.0 \times 10^{-21} \mathrm{~J}$, and the CCC in $z=2$ might be $>80 \mathrm{mM}$. This estimation did not match with the results of measurement of the DLS, which indicated strong aggregation of the dendrimer particles in the presence of even $1.0 \mathrm{mM}$ of $\mathrm{Ca}^{2+}$.

\section{AFM imaging of the dendrimer aggregates}

To investigate existing forms of dendrimer particles in a culture medium, an AFM observation was performed in liquid. 


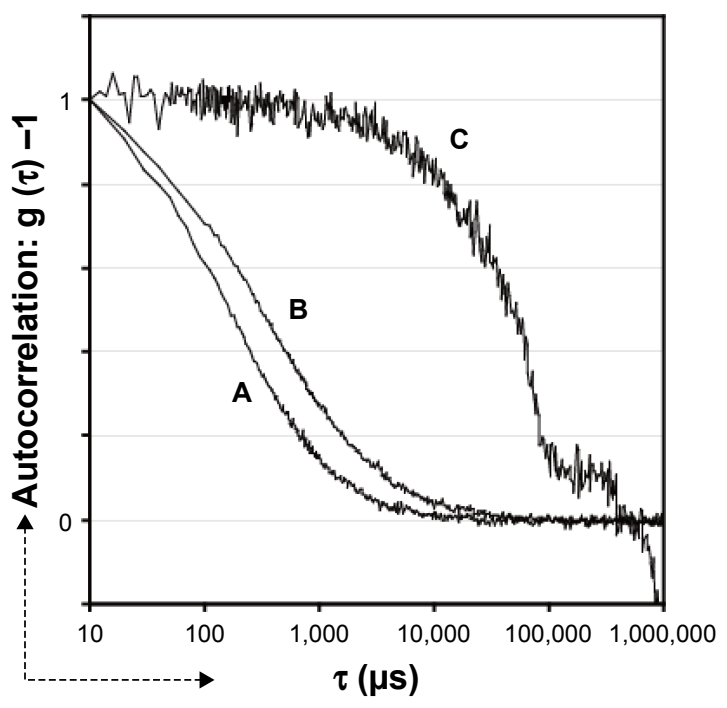

Figure 4 Autocorrelation curves determined by fluorescence correlation spectroscopy.

Notes: Alexa 546-labeled cationic PAMAM dendrimers were suspended in the following solutions: (A) $\mathrm{H}_{2} \mathrm{O}$; (B) PBS (-) with $\mathrm{HSA}(50 \mathrm{mg} / \mathrm{mL}) ;($ C) PBS $(+)$ with HSA $(50 \mathrm{mg} / \mathrm{mL})$. The three curves obtained were superimposed after adjustment for the autocorrelation values at $\tau=10 \mu \mathrm{s}$.

Abbreviations: HSA, human serum albumin; PAMAM, polyamidoamine; PBS(-), phosphate buffered saline without calcium/magnesium; PBS(+), phosphate buffered saline with calcium/magnesium.

Immediately after putting a drop of the dendrimer suspension in DMEM onto a freshly cleaved mica plate, the plate was scanned with dynamic mode in DMEM. Several spheroidal particles with diameter of 20-60 nm were detected (Figure 5), and they were considered as the dendrimer nanoparticles that had aggregated, floated, and finally adhered to the mica plate

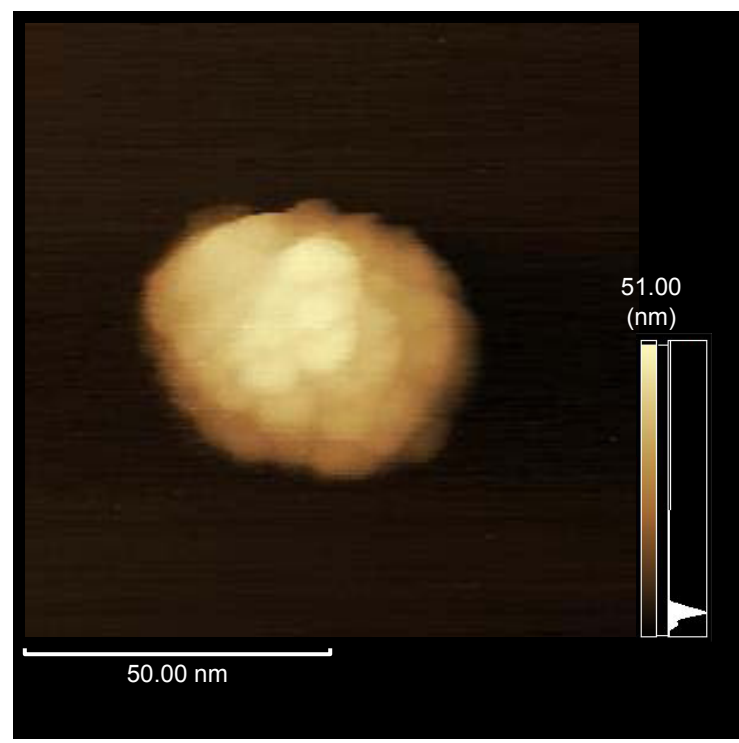

$100.00 \times 100.00 \mathrm{~nm}$

Figure 5 AFM image of the aggregate of PAMAM dendrimer nanoparticles on the mica plate in DMEM.

Note: AFM observation was performed at scan rate of $2.0 \mathrm{~Hz}$ in a dynamic mode with drive frequency of $20 \mathrm{kHz}$.

Abbreviations: AFM, atomic force microscopy; DMEM, Dulbecco's Modified Eagle's Medium; PAMAM, polyamidoamine. electrostatically. The result provided evidence that prompt aggregation of the dendrimer particles occurs in the liquid equivalent to body fluids.

\section{Discussion}

In this study, SPECT imaging of a mouse failed to reveal any radioactive signal in the intracranial region at 1,3 , or 7 hours after intravenous injection of the cationic PAMAM dendrimer particles. Signals were focused on the liver, spleen, and kidney, consistent with the findings of previous studies. Malik et al reported that at 1 hour after intravenous injection, $30 \%-90 \%$ of cationic ${ }^{125}$ I-labeled PAMAM dendrimer particles (G3, G4) were distributed in the liver. ${ }^{19}$ Nigavekar et al reported that after intravenous injection of partially acetylated cationic ${ }^{3} \mathrm{H}$ PAMAM dendrimer particles (G5), the highest uptakes were observed in the lungs, kidney, and liver, and the least uptake was observed in the brain. ${ }^{20}$ Zhang et al reported the results of planar Y-camera imaging of ${ }^{125}$ I-labeled PAMAM dendrimer particles (G4), which demonstrated predominant accumulation in the liver, kidney, and spleen, but very limited detection in the brain. ${ }^{21}$ Our results lent support to the notion that after intravenous injection, cationic dendrimer particles are scarcely distributed in the cerebral region. Specific distribution of dendrimer particles in the kidney, liver, and spleen indicated their process of removal from the blood circulation via renal glomerular filtration and trapping by the RES. This process involves a simple leak through capillaries, which have pores or clefts, the so-called fenestrated or sinusoidal capillaries, and is an inevitable fate for a large amount of circulating particles. Only particulate that can escape this process of elimination and can flow into continuous capillaries in general tissues, including the brain, can attain transference to the interstitial regions by transcytosis through endothelial cells.

Cationic dendrimer particles can easily attach themselves to cell membranes with a negative charge and be promptly internalized via the process of cellular pinocytosis. In vitro studies carried out by us and others have demonstrated that cationic PAMAM dendrimer particles are rapidly captured by cultured cells and trapped within endosomal vesicles. ${ }^{10,13}$ As PAMAM dendrimers are considered to have no counteracting antibodies, receptors, or transporter molecules on the cell surface, the main mechanism for BBB permeation of these particles would be adhesion-induced cellular pinocytosis and succeeding exocytosis, the so-called process of adhesive transcytosis..$^{22,23}$ In this study, we measured the in vitro BBB permeability of dendrimer nanoparticles, which revealed a permeability coefficient of $8.0 \times 10^{-6} \mathrm{~cm} / \mathrm{s}$; this value was considered to indicate poor BBB permeation 
in comparison with that reported by previous studies. ${ }^{24-26}$ In another experiment, we performed the same test with dendrimer nanoparticles suspended in human serum instead of in the assay buffer and obtained very similar results (data not shown). These results suggest that low BBB permeability in vivo of cationic PAMAM dendrimer particles is unexpected because of prompt cellular internalization of the dendrimer particles in cultured cells as we observed. These discrepant findings may be attributable, as we argue later, to the material properties of cationic dendrimers, their propensity for easy aggregation.

Measurement of the DLS in our study demonstrated that when the dendrimer particles were suspended in a solution of electrolytes having a similar composition to that of body fluids, they aggregated promptly to form the aggregates of submicron- or larger sizes. AFM images of a freshly cleaved mica plate soaked in a dendrimer suspension (DMEM) confirmed prompt aggregation. In the DLS experiments using dispersion media containing various concentrations of electrolytes, we observed that prompt aggregation occurred in the presence of $\mathrm{Ca}^{2+}$ even at low concentration, but not in the presence of $\mathrm{Na}^{+}$even at high concentrations. The results of our physicochemical analysis revealed that such prompt aggregation of dendrimers cannot be explained by the DLVO theory, which is established as a highly effective theory for explaining the dispersive modality of colloids and nanoparticles. ${ }^{27}$

Our data indicated that the potential for the aggregation of dendrimers was not associated with the concentrations of univalent cations in the dispersal medium, but was related to the concentration of divalent cations. This possible effect of divalent cations cannot be explained by the DLVO theory and is, thus, probably attributable to the so-called "non-DLVO force". Although calcium can induce aggregation of some nanomaterials, such as liposomes, ${ }^{28}$ there has been no previous report demonstrating a similar phenomenon for the case of dendrimers, and unfortunately, we could not characterize the force in further detail in this study.

General body fluids contain components other than electrolytes that can influence the dispersive state of intravenously administered agents. Albumin is one of the major components of body fluids and is supposed to be adsorbed onto various bioactive materials as a carrier or a stabilizer of other protein molecules. Several basic studies have suggested that albumin molecules can be adsorbed onto the surfaces of nanomaterials, preventing their aggregation. ${ }^{29} \mathrm{In}$ the case of dendrimers, previous studies have demonstrated that a few molecules of dendrimers and one molecule of albumin form an assembly, which is sustained for a long period of time..$^{30,31}$ These reports partially lend support to the results of our initial FCS, which indicated that cationic dendrimers and HSA formed 1:1 assemblies in PBS (-). Our further FCS analysis, however, revealed that mixing of HSA in PBS (+) led to the formation of dendrimer aggregates of submicron- or larger sizes. After these examinations, we concluded that cationic PAMAM dendrimers in the plasma tend to show marked aggregation despite the coexistent albumin molecules.

On the basis of the above results, the possibility arises, in relation to the results of the BBB permeation test, that the marked aggregation of the dendrimer particles occurring in the medium in the apical aspect of the BBB layer influences the degree of translocation to the basolateral aspect. The cellular uptake of particulate materials occurs by endocytosis in the case of nano-sized particles and by macropinocytosis in the case of larger particles. ${ }^{23}$ In our test, judging from the observed effects of the cellular uptake inhibitors, the dendrimer particles permeating across the endothelial cells comprising the BBB must have been transferred by the process of endocytosis-exocytosis, not by that of macropinocytosis-exocytosis. In general, the mechanism of cell internalization of the aggregates shifts, as the aggregation proceeds, from endocytosis to micropinocytosis, with the latter being scarcely linked to exocytosis, and the former being commonly linked to exocytosis. Therefore, it is supposed that the factors promoting aggregation suppress the BBB permeation of the formed aggregates. ${ }^{32}$

\section{Conclusion}

Our findings suggest that amine-terminated cationic PAMAM dendrimer particles aggregate promptly upon mixing with body fluids, form submicron-sized to larger aggregates. Large-sized particles may be trapped and engulfed by cells via macropinocytosis rather than by endocytosis, making transcytosis across the endothelial cells comprising the BBB difficult. Furthermore, the rather low rate of transport of intravenously administered dendrimer particles into the intracranial nervous tissue appears to be due mainly to their propensity to prompt aggregation. For the utilization of cationic PAMAM dendrimer particles as effective nanodevices for drug delivery into brain tissue, we must find to reduce the propensity of the dendrimer particles for aggregation upon mixing with body fluids.

\section{Acknowledgments}

This study was supported by a Grant-in-Aid for Scientific Research from the Ministry of Education, Culture and 
Science (No 24241013) and by a Project Grant from National Institute for Environmental Studies (NIES) (No 13230).

\section{Disclosure}

The authors report no conflicts of interest in this work.

\section{References}

1. Labuzek K, Gorki K, Jaroszek H, Jarzabek K, Gabryel B, Okopien B. Highly organized nanostructures for brain drug delivery-new hope or just a fad? CNS Neurol Disord Drug Targets. 2015;12(8):1271-1285.

2. Agarwal A, Lariya N, Saraogi G, Dubey N, Agrawal H, Agrawal GP. Nanoparticles as novel carrier for brain delivery: a review. Cur Pharm Design. 2009;15(8):917-925.

3. Rubin LL, Staddon JM. The cell biology of the blood-brain barrier. Annu Rev Neurosci. 1999;22:11-28.

4. Klajnert B, Bryszewska M. Dendrimers: properties and applications. Acta Biochim Pol. 2001;48(1):199-208.

5. Svenson S, Tomalia DA. Dendrimers in biological applications-reflections on the field. Adv Drug Deliv Rev. 2005;57(15):2106-2129.

6. Wu H, Huang W, He Z. Dendrimers as carriers for siRNA delivery and gene silencing: a review. Sci World J. 2013;2013:630654.

7. Xu L, Zhang $\mathrm{H}, \mathrm{Wu}$ Y. Dendrimer advances for the central nervous system delivery of therapeutics. ACS Chem Neurosci. 2014;5(1):2-13.

8. Kobayashi H, Kawamoto S, Saga T, et al. Novel liver macromolecular MR contrast agent with a polypropylenimine diaminobutyl dendrimer core: comparison to the vascular MR contrast agent with the polyamidoamine dendrimer core. Magn Reson Med. 2001;46(4):795-802.

9. Tomalia DA, Baker H, Dewald J, et al. A new class of polymersstarburst-dendritic macromolecules. Polym J. 1985;17:117-132.

10. Adjei IM, Sharma B, Labhasetwar V. Nanoparticles: cellular uptake and cytotoxicity. Adv Exp Med Biol. 2014;811:73-91.

11. Boussif O, Lezoualch F, Zanta MA, et al. A versatile vector for gene and oligonucleotide transfer into cells in culture and in vivo: polyethylenimine. Proc Natl Acad Sci U S A. 1995;92(16):7297-7301.

12. Freeman EC, Weiland LM, Meng WS. Modeling the proton sponge hypothesis: examining proton sponge effectiveness for enhancing intracellular gene delivery through multiscale modeling. $J$ Biomater Sci Polym Ed. 2013;24(4):398-416.

13. Zeng Y, Kurokawa Y, Win-Shwe TT, et al. Effects of PAMAM dendrimers with various surface functional groups and multiple generations on cytotoxicity and neuronal differentiation using human neural progenitor cells. J Toxicol Sci. 2016;41(3):351-370.

14. Watala ML, Watala C. PAMAM dendrimers: destined for success or doomed to fail? plain and modified PAMAM dendrimers in the context of biomedical applications. J Pharm Sci. 2015;104(1):2-14.

15. Kojima C, Regino C, Umeda Y, Kobayashi H, Kono K. Influence of dendrimer generation and polyethylene glycol length on the biodistribution of PEGylated dendrimers. Int J Pharm. 2010;383(1-2):293-296.

16. Naha PC, Davoren M, Lyng FM, Byrne HJ. Reactive oxygen species (ROS) induced cytokine production and cytotoxicity of PAMAM dendrimers in J774A.1 cells. Toxicol Appl Pharmacol. 2010;246(1-2): 91-99.
17. Nyitrai G, Heja L, Jablonkai I, Pal I, Visy J, Kardos J. Polyamidoamine dendrimer impairs mitochondrial oxidation in brain tissue. J Nanobiotech. 2013;11:9.

18. Vaidyanathan G, Zalutsky MR. Preparation of N-succinimidyl 3-[*I] iodobenzoate: an agent for the indirect radioiodination of proteins. Nat Protoc. 2006;1(2):707-713.

19. Malik N, Wiwattanapatapee R, Klopsch R, et al. Dendrimers: relationship between structure and biocompatibility in vitro, and preliminary studies on the biodistribution of 125I-labelled polyamidoamine dendrimers in vivo. J Control Release. 2000;65(1-2):133-148.

20. Nigavekar SS, Sung LY, Llanes M, et al. ${ }^{3} \mathrm{H}$ dendrimer nanoparticle organ/tumor distribution. Pharm Res. 2004;21(3):476-483.

21. Zhang Y, Sun Y, Xu X, et al. Synthesis, biodistribution, and microsingle photon emission computed tomography (SPECT) imaging study of technetium-99m labeled PEGylated dendrimer poly (amidoamine) (PAMAM)-folic acid conjugates. J Med Chem. 2010; 53(8):3262-3272.

22. Tuma PL, Hubbard AL. Transcytosis: crossing cellular barriers. Physiol Rev. 2003;83(3):871-932.

23. Hillaiceau H, Couvreur P. Nanocarriers' entry into the cell: relevance to drug delivery. Cell Mol Life Sci. 2009;66(17):2873-2896.

24. Gaillard PJ, de Boer AG. Relationship between permeability status of the blood-brain barrier and in vitro permeability coefficient of a drug. Eur J Pharm Sci. 2000;12(2):95-102.

25. Nakagawa S, Deli MA, Kawaguchi H, et al. A new blood-brain barrier model using primary rat brain endothelial cells, pericytes and astrocytes. Neurochem Int. 2009;54(3-4):253-263.

26. Hanada S, Fujioka K, Inoue Y, Kanaya F, Manome Y, Yamamoto K. Cell-based in vitro blood-brain barrier model can rapidly evaluate nanoparticles' brain permeability in association with particle size and surface modification. Int J Mol Sci. 2014;15(2):1812-1825.

27. Israelachvili JN. Intermolecular and Surface Forces. 3rd ed. Amsterdam, NL: Elsevier; 2011.

28. Wilschut J, Duzgunes N, Fraley R, Papahadjopoulos D. Studies on the mechanism of membrane fusion: kinetics of calcium induced fusion of phosphatidylserine vesicles followed by a new assay for mixing of aqueous vesicle contents. Biochemistry. 1980;19(26):6011-6021.

29. Bhirde AA, Hassan SA, Harr E, Chen X. Role of albumin in the formation and stabilization of nanoparticle aggregation in serum studied by continuous photon correlation spectroscopy and multiscale computer simulations. J Phys Chem C Nanomater Interfaces. 2014;118(29): 16199-16208.

30. Shcharbin D, Janicka M, Wasiak M, et al. Serum albumins have five sites for binding of cationic dendrimers. Biochim Biophys Acta. 2007;1774(7):946-951.

31. Giri J, Diallo MS, Simpson AJ, et al. Intercations of poly(amidoamine) dendrimers with human serum albumin: binding constants and mechanisms. ACS Nano. 2011;5(5):3456-3468.

32. Chithrani BD, Chen WCW. Elucidating the mechanism of cellular uptake and removal of protein-coated gold nanaoparticles of different sizes and shapes. Nano Lett. 2007;7(6):1542-1550.
International Journal of Nanomedicine

\section{Publish your work in this journal}

The International Journal of Nanomedicine is an international, peerreviewed journal focusing on the application of nanotechnology in diagnostics, therapeutics, and drug delivery systems throughout the biomedical field. This journal is indexed on PubMed Central, MedLine, CAS, SciSearch ${ }^{\circledR}$, Current Contents ${ }^{\circledR} /$ Clinical Medicine,

\section{Dovepress}

Journal Citation Reports/Science Edition, EMBase, Scopus and the Elsevier Bibliographic databases. The manuscript management system is completely online and includes a very quick and fair peer-review system, which is all easy to use. Visit http://www.dovepress.com/ testimonials.php to read real quotes from published authors. 